\title{
LA TRIDIMENSIONALIDAD DEL CONCEPTO DE NUTRICIÓN: SU RELACIÓN CON LA EDUCACIÓN PARA LA SALUD
}

\section{THE THREE-DIMENSIONALITY OF THE NUTRITION CONCEPT: ITS RELATIONSHIP WITH HEALTH EDUCATION}

\author{
Adriana Ivette Macias M., María Luisa Quintero S., \\ Esteban Jaime Camacho R., Juan Manuel Sánchez S. \\ Unidad Académica Profesional Nezahualcóyotl, Universidad Autónoma del Estado de México, México.
}

\begin{abstract}
The evolution of the nutrition concept is described until arriving to the concept of three-dimensionality, analyzing it from at the world level, in Latin America and Mexico. Nutrition used to be considered an exclusively biological process; nevertheless at the moment it involves social and ecological aspects. The convergence between the health concept and nutrition from the biosocial conception of the individual is analyzed, emphasizing the importance of education as a promotional tool which includes not only includes the informative aspect but also the modification of behavior to change the individual's lifestyle.
\end{abstract}

Key words: health, health promotion, chronic diseases, community nutrition, nutrition education.

Este trabajo fue recibido el 19 de Enero de 2009 y aceptado para ser publicado el 20 de Septiembre de 2009.

\section{INTRODUCCIÓN}

La presente revisión del concepto de nutrición y su articulación con la educación para la salud se deriva ante la problemática de considerar a la nutrición como un aspecto netamente biológico, lo que limita a las acciones dirigidas a mejorar la situación nutricional de la población actual. Con la nueva tridimensionalidad de la nutrición se pretende modificar la concepción del concepto al integrar los aspectos sociales y ecológicos que intervienen en esta. En cuanto a la convergencia con la educación para la salud, reside en que esta última al ser de carácter multidisciplinario distingue al ser humano y a sus problemáticas de salud desde una perspectiva integral que involucra el contexto biológico, social y ambiental.

Por otro lado el objetivo de dicha revisión permite establecer la tridimensionalidad de la nutrición como un determinante que interviene en la salud individual y colectiva, si se considera que en la actualidad las enfermedades crónicas - degenerativas no solo se relacionan con desequilibrios metabólicos ocasionados por una inadecuada educación nutricional sino también por aspectos sociales y medio ambientales, por lo que es importante utilizar a la educación para la salud como una medio preventivo y promocional, que contribuya a la adopción de hábitos alimentarios adecuándolos a los aspectos biológicos, sociales y medio ambientales de cada comunidad.

En base a lo anterior vivimos en un mundo cambiante en el que a cada segundo se generan nuevos conocimientos, gracias a los avances tecnológicos y a los descubrimientos en las ciencias, por lo que la salud no es la excepción. Un claro ejemplo es la "nueva ciencia de la nutrición" (1) que se deriva de un proceso evolutivo del concepto de nutrición a lo largo del siglo XX y principios del siglo XXI, desde la fundación de la Organización Mundial de la Salud en 1948 hasta la Declaración de Giessen en el 2005, la nutrición en sus inicios era concebida exclusivamente como un proceso biológico esa evolución ha propiciado que actualmente sea considerada como una ciencia multidisciplinaria y de gran complejidad. Estos cambios han sido marcados de manera conjunta con los avances y descubrimientos en la industria alimentaria que atiende principalmente 
a intereses económicos. Por otro lado, el cambio en los estilos de vida y la adopción de nuevos hábitos alimentarios han propiciado una evolución acelerada del concepto de nutrición a nivel internacional, continental (América Latina) y nacional.

En la actualidad la incidencia de enfermedades crónicas ha ido en aumento, por lo que resulta necesaria la búsqueda de herramientas que tomen como base la tridimensionalidad del concepto de nutrición para informar y proporcionar a las poblaciones los conocimientos precisos para la adopción de estilos de vida saludables, objetivo que sólo se logrará mediante la educación para la salud.

La tridimensionalidad del concepto de nutrición abarca la dimensión biológica, como un proceso indispensable para el mantenimiento de la vida, la dimensión social en el que intervienen factores culturales tales como la religión, educación y hábitos alimentarios, así como factores económicos. La última dimensión abarcada en la tridimensionalidad es la ambiental en la que se menciona la importancia de la sustentabilidad de alimentos y la utilización de cultivos como fuente de energía como medidas que no solo apoyan la parte nutricional de las poblaciones sino que también contribuyen a la conservación del medio ambiente.

\section{EVOLUCIÓN DEL CONCEPTO DE NUTRICIÓN}

\section{Inicios de los estudios de nutrición y las guerras mundiales}

Definir a la nutrición no es cosa sencilla ya que implica trasladarnos hasta los inicios de la humanidad cuando el hombre ingería alimentos solo para sustento. Los primeros estudios científicos de la nutrición se realizaron en Europa durante el siglo XIX y en ellos quedaron establecidos los principios fundamentales del concepto de la nutrición como un proceso utilizado por el hombre para obtener energía. A partir de aquí y hasta el término de la Segunda Guerra Mundial, los avances en el concepto de la nutrición van a estar enfocados al descubrimiento de los nutrientes que actualmente conocemos como macromoléculas o macronutrientes (proteínas, carbohidratos, lípidos y vitaminas) (2).

Al terminar la Segunda Guerra Mundial se crearon dos organismos internacionales para atender, de manera conjunta, los problemas ocasionados (por la guerra) en materia de salud, (incluyendo los problemas alimentarios): la FAO (Organización para la Alimentación y Agricultura, sus siglas en inglés) en 1943 y la OMS (Organización Mundial de la Salud) en 1948; esta última define a la nutrición como "un conjunto de procesos mediante el cual los seres vivos incorporan, modifican y eliminan sustancias procedentes del exterior" (2). De manera generalizada, estos son los inicios de una nueva ciencia, pero mencionaremos algunos otros conceptos que nos permitirán entender mejor la evolución de este concepto hasta llegar a una complejidad en el término y que en muchas de ellas entra en controversia, pero que finalmente se llega a la tridimensionalidad del nuevo concepto.

\section{La nutrición durante el siglo XX}

A mediados del siglo XX el Ministerio de Agricultura de la Gran Bretaña considera que la ciencia de la nutrición exige el estudio de todos los procesos del crecimiento, mantenimiento y reparación del cuerpo vivo que dependen de los alimentos (3); mientras tanto, en España, Francia y Alemania la nutrición es considerada como una función biológica, una disciplina o una ciencia desconocida de reciente creación (3).

\section{Concepciones actuales de la nutrición: siglo XXI}

A principios del siglo XXI, en el $17^{\circ}$ Congreso Internacional de Nutrición en Viena, (2001) se define a la nutrición como el estudio de la totalidad de la relación entre las características funcionales del organismo (comportamiento metabólico) y su medio ambiente, dando énfasis al aporte calórico que proporcionan los alimentos, así como la importancia de una dieta (3). En 2005, durante la Declaración de Giessen, se propone la tridimensionalidad del concepto de la nueva ciencia de la nutrición la cual es definida como el estudio de los alimentos y bebidas así como la constitución de otros alimentos y de las interacciones con los sistemas biológicos, sociales y ambientales más relevantes (4). De acuerdo con la concepción Iberoamericana la nutrición es un amplio y complejo conjunto de fenómenos biológicos, psicoemocionales y socioculturales asociados con la obtención, asimilación y metabolismos de los nutrientes, por lo que su estudio es necesariamente interdisciplinario concurriendo en ella la biología, ecología, historia, política, economía, psicología, antropología, sociología y cualquier disciplina que aborde factores que afecten a la nutrición (5). Aquí es donde radica su multidisciplinariedad, pero se debe tener en cuenta que, aunque es un proceso individual, con la introducción de este nuevo concepto se vuelve social y se ve afectado de manera particular en cada país del globo terráqueo, debido a los cambios socioeconómicos y políticos que sufre cada nación.

\section{La nutrición en América Latina}

En los años 30 y 40 tanto en Europa como en América Latina los estudios de nutrición están marcados 
por la preocupación de definir la dieta normal para las poblaciones. En los años 20 surge en América Latina la figura de Pedro Escudero que tuvo una gran influencia en los estudios de nutrición, durante una investigación sobre Bolivia en 1947 señaló que el problema alimentario de un pueblo presenta 3 fases: la primera biológica, la segunda económica y la tercera cultural.

En 1943 durante la Conferencia en Hot Spring, se atendió la preocupación de crear un organismo que tratara los problemas de alimentación y agricultura, este hecho originó la creación de la FAO. A partir de dicha conferencia efectuada en Estados Unidos de América, se da la pauta para que en América Latina se den cuatro conferencias de nutrición (1948-1956) en la que participaron organizaciones de índole internacional como la OMS y la FAO que permitieron el encuentro de especialistas en nutrición para discutir problemas y proponer soluciones a las problemáticas de la región, que posteriormente antecedieron a la creación de la Sociedad Latinoamericana de Nutrición (SLAN) en 1964 (6).

\section{La nutrición en México}

En México, los inicios de la nutrición tienen su origen en Mesoamérica con el desarrollo de la agricultura, donde las culturas mesoamericanas tenían grandes bancos de alimentos que abastecían a toda la población. Cabe destacar que esta repartición era de manera inequitativa, donde únicamente las clases sociales privilegiadas tenían acceso a los mejores alimentos y por ende a una buena nutrición.

Con la colonización, el choque entre dos culturas provocó la estructuración de una sociedad desigual donde grandes sectores de la población vivían en condiciones miserables de ignorancia y pobreza, por lo que su poder adquisitivo para obtener alimentos que cubrieran sus necesidades nutrimentales básicas era imposible, por lo que se produce una ola de desnutrición endémica.

Casi un siglo después de la Independencia mexicana, José Patrón Correa publicó en 1908 en la Revista Médica de Yucatán un artículo sobre "la culebrilla" (nombre que se le daba a la desnutrición infantil en el sureste de México, y que se caracterizaba por la descamación de la piel, similar a la muda de piel de muchas serpientes). A partir de este hecho, se originaron investigaciones sobre las propiedades nutricionales de muchos alimentos, pero fue hasta 1943 que se creó el Instituto Nacional de Nutriología (INNu) que después cambiará su nombre en 1980 a Instituto Nacional de Nutrición Salvador Zubirán (INNSZ), convirtiéndose actualmente en el órgano nacional más importante en cuanto a investigación en materia de nutrición (6).

\section{LA DIMENSIÓN BIOLÓGICA}

La clásica concepción acerca de la nutrición como un "proceso biológico", sigue siendo el eje central de la nueva ciencia de la nutrición que en sus inicios solo era concebida como un proceso mediante el cual el ser humano transformaba los alimentos que consumía con el único fin de obtener energía y así poder realizar sus actividades diarias, pero así como la nutrición ha tenido una evolución en su concepto, cada una de las dimensiones que actualmente la conforman han sufrido de igual manera considerables modificaciones. Partiendo de esta afirmación en el ámbito biológico la nutrición ha evolucionado, tal como se muestra a continuación.

Se mencionan dos conceptos que engloban la noción actual de la nutrición a nivel biológico, el primero de ellos es: "conjunto de procesos mediante los cuales nuestro organismo, utiliza transforma e incorpora a sus propios tejidos una serie de sustancias que recibe del mundo exterior y que han de cumplir tres fines básicos: aportar la energía necesaria para mantener la integridad y el funcionamiento de las estructuras corporales, construir y reparar estas estructuras y regular los procesos metabólicos" (7). Por otro lado para Grande, "es el conjunto de procesos mediante los cuales el hombre ingiere, absorbe, transforma y utiliza las sustancias que se encuentran en los alimentos y que tienen que cumplir cuatro importantes objetivos: suministrar energía para el mantenimiento de sus funciones y actividades, aportar materiales para la formación, crecimiento y reparación de las estructuras corporales y para la reproducción, suministrar las sustancias necesarias para regular los procesos metabólicos y reducir el riesgo de algunas enfermedades" (8).

Aunque ambas definiciones parecen ser similares en cuanto a la concepción y funciones de la nutrición, cabe destacar que en esta última se agrega una función más, "reducir el riesgo de algunas enfermedades" y que es de suma importancia en la actualidad, ya que debido al creciente ritmo de vida han ido en aumento las enfermedades crónico degenerativas, tales como la diabetes, obesidad, hipertensión arterial y las enfermedades cardiovasculares que en la mayoría tienen como causa un estilo de vida inadecuado. Sin embargo cabe destacar que aunque la nutrición juega un papel importante, por si sola no disminuye dichas enfermedades, si es un determinante que ayuda a prevenirlas desde sus inicios y así lograr una buena salud. La nutrición por lo tanto es un proceso a nivel celular que depende de las características metabólicas del individuo.

\section{LA DIMENSIÓN SOCIAL}

"En casi todos los países, los factores sociales y culturales tienen un influencia muy grande sobre lo que 
come la gente, cómo preparan sus alimentos, sus prácticas alimentarias y los alimentos que prefieren" (9). En este sentido intervienen determinantes sociales, culturales (religión y educación) y económicos que afectan a la nutrición de manera directa e indirecta. Por otro lado en la nueva propuesta de la dimensión social se menciona que la nutrición va de prácticas individuales a poblacionales surgiendo así el término de nutrición comunitaria (10).

Todos los países tienen una cultura alimentaria en la que se ven inmersos la elección de alimentos y los hábitos, en muchas de las ocasiones esta cultura alimentaria limita la ingesta de algunos nutrientes que el cuerpo necesita, debido a los tabúes o a los malos hábitos. Por ejemplo en África se cree que si las mujeres consumen huevo pueden quedar estériles, sin embargo esto no es cierto y además limita a las mujeres a obtener la albúmina. Aunque se cree que los hábitos nunca cambian o son difíciles de modificar, eso no es tan cierto ya que los hábitos no son los mismos que hace unos años lo que se debe generalmente a cambios sociales y económicos. Por otro lado la religión juega un papel importante en la nutrición de los individuos, ya que algunas religiones limitan la ingesta de ciertos alimentos lo que conlleva a no ingerir los nutrientes necesarios que proporcionen un estado nutricional normal. Cada país tiene características económicas diferentes, por lo que el nivel adquisitivo es inequitativo y mientras en un país prevalecen enfermedades relacionadas a la baja obtención de nutrientes, en otros existen altos índices de enfermedades crónicas por la alta ingesta de grasas saturadas.

Por tanto, la nutrición comunitaria es definida como "el conjunto de actividades vinculadas a la salud pública que dentro del marco de la nutrición aplicada se desarrollan con un enfoque participativo de la comunidad" (10). Este enfoque nos permite establecer la sustentabilidad que debe de existir por los miembros de una comunidad para satisfacer sus necesidades nutrimentales, por lo que el futuro de esta nueva ciencia dependerá de marcos sociopolíticos, culturales y económicos.

Cabe destacar que nuevamente en esta dimensión se menciona a la salud como un elemento importante en la concepción actual de la nutrición, "en el mundo moderno, las fronteras fácilmente cruzadas por las enfermedades..., la carga social cada vez mayor de enfermedades crónicas, los factores económicos, sociales y políticos, repercuten en la salud humana" (11).

\section{LA DIMENSIÓN AMBIENTAL}

La población humana ha aumentado y las consecuencias en el medio ambiente cada vez son más graves, dichas modificaciones repercuten en la producción de alimentos ya que en algunas zonas del planeta el curso de las estaciones de año se ha modificado provocando largos períodos de sequías o bien lluvias exageradas que consiguen que se pierdan grandes hectáreas de sembradíos. Aunque la ciencia de la nutrición ha tenido grandes avances no ha sido de igual manera entre las distintas dimensiones, tal es el caso de esta, por lo que la nueva tendencia a seguir en esta perspectiva es "asegurarse de que la práctica de nuestra ciencia apoye ecosistemas sostenibles y ambientes sanos" y así influir en uno de los determinantes de la salud: el medio ambiente (12).

En la actualidad la disponibilidad de alimentos se ha visto afectada por las modificaciones en el medio ambiente, así la erosión de la tierra ha provocado que en muchos lugares del planeta no se cultiven alimentos que con anterioridad era muy frecuente encontrarlos, es aquí donde interviene la nueva nutrición sostenible que implica que sea ecológicamente sustentable, muestra de ello es la producción de pescados, de un arroz más nutritivo, así como frutas y verduras, entre otros (12). Por otro lado ante el posible agotamiento del petróleo como principal fuente de energía se han buscado alternativas como los biocombustibles que se obtienen a través de azucares, almidones y celulosa de algunos cultivos como la caña de azúcar, maíz, sorgo, remolacha y soya. Si se considera que se sufrirá de una escases de granos, esto implicaría una baja disponibilidad de granos para la alimentación de las poblaciones (13).

Pero los avances en ciencia y tecnología no serán suficientes para resolver los problemas gigantescos de preservar los recursos naturales y humanos y así proporcionar las respuestas relacionadas con las ediciones globales tales como la influencia económica/política en suministro de alimentos y la nutrición adecuada (11). La sustentabilidad de la nutrición va a depender de la cooperación de las estrategias empleadas en todos los países, para lograr con esto que los alimentos estén distribuidos de manera equitativa en todo el planeta, en este sentido ha aparecido la denominada ecología de la nutrición que se define como "el conjunto de los sistemas de la nutrición que recomiendan una nutrición sana como la manera más conveniente y sostenible de comer y beber, lo que conlleva a promover una dieta saludable, equitativa y sostenible" (14).

\section{EDUCACIÓN PARA LA SALUD}

En la Conferencia Internacional de Promoción de la Salud de Ottawa en 1986 organizada por la OMS se aprobó la Carta de Ottawa en la que se establecen las bases de la doctrina de la Promoción para la Salud lo que implica pasar de lo teórico a lo práctico y que además se convierte en la herramienta para lograr la estrategia de la OMS sobre la Atención Primaria de Salud en Alma Ata 
(1977) “Salud para todos en el año 2000”. De acuerdo con este documento la Promoción para la Salud consiste en proporcionar a la gente los medios necesarios para mejorar su salud y alcanzar un mayor control sobre la misma (15). Fue hasta 1990 cuando en América Latina se incluyó la Promoción de la Salud como componente de las políticas nacionales de salud.

Acorde con la definición de la Promoción de la Salud en proporcionar los medios necesarios para mejorar la salud, surge una nueva interrogante ¿Cómo facilitar dichos medios?; una solución a esta incógnita fue el desarrollo de una nueva disciplina combinando a las ciencias de la Educación y la Salud, dando como resultado el término de la Educación para la Salud (EpS). Aunque no se tiene una fecha exacta de cuando empezó esta disciplina, en la $36^{\text {a }}$ Asamblea Mundial de la Salud se define a la EpS como cualquier combinación de actividades de información y educación que lleve a una situación en la que la gente sepa cómo alcanzar la salud y busque ayuda cuando lo necesite (16).

Cabe destacar que en el desarrollo de esta disciplina se dieron lugar dos enfoques de la EpS; el primero se caracterizó por ser informativo prescriptivo, el cual implicaba un proceso de transmisión de información con una intención preceptiva que consistía en orientar actividades educativas y en donde los profesionales de la salud son los únicos que poseían los conocimientos; el segundo enfoque surgió como producto de la preocupación de la medicina por la conducta y sus resultado en la salud, en este sentido se concebía a la EpS como una serie de intervenciones destinadas a facilitar cambios en la conducta y en los estilos de vida cuyo objetivo era conseguir comportamientos saludables, ambos enfoques dieron lugar a la concepción de la EpS como cualquier esfuerzo de proporcionar información y conocimientos relativos al mantenimiento y Promoción de la Salud (17). En este sentido la EpS constituye uno de los medios más efectivos para modificar creencias, costumbres y hábitos negativos de la comunidad, además de proporcionar orientación a la sociedad en relación con los medios para promover, proteger y fomentar la salud. El campo de la EpS es muy amplio y dentro de el esta incluido la nutrición (18). Aunque la EpS tiene una función preventiva y correctiva sus principales propósitos son la prevención de enfermedades tanto transmisibles como no transmisible (crónicas) y la promoción de estilos de vida saludables, que van a dar como consecuencia una buena calidad de vida (16).

\section{DISCUSIÓN}

Si bien el concepto de nutrición no es nuevo, la tridimensionalidad del mismo sí y se debe de considerar que esta evolución ha ido a la par con la consideración holística de la salud, al ser la nutrición un determinante de la salud en los individuos y presenta una interacción en el origen de las enfermedades crónicas. Cabe destacar que al igual que en la salud, intervienen distintos factores que influyen en la nutrición que no siempre dependen del individuo sino que ahora también de la comunidad, como sucede con las dimensiones de la nutrición que van de un nivel macro a micro y de lo colectivo a lo individual, debido a que la dimensión ambiental depende de la nutrición sustentable que es gestionada por las políticas nacionales y que en conjunto se busca la cooperación internacional, por otra parte la dimensión social depende esencialmente de los patrones y normas de cultura que establecen los grupos sociales a los que se pertenecen y finalmente la dimensión biológica depende exclusivamente del individuo y de sus características metabólicas y fisiológicas del mismo. Debemos de considerar que hablar de nutrición nos remite indudablemente a pensar en la alimentación que es la manera en como adquirimos los alimentos que nos proporcionan los nutrientes necesarios que nos dan el aporte energético, por lo que es inevitable incluir a esta nueva perspectiva de la nutrición un elemento importante la "promoción y educación en materia de nutrición" ya que en las tres dimensiones mencionadas se habla de reducir riesgos de algunas enfermedades mediante estrategias específicas como la sustentabilidad, es por esto que una nueva estrategia a involucrar es la educación para la salud, que no sólo va a tener como meta el informar si no también el de prevenir enfermedades crónicas generalmente provocadas por una mala nutrición.

Otro punto que debe de recatarse es la comparación que existe entre la tridimensionalidad del término de salud con el de nutrición, ya que por un lado, la salud involucra lo biológico, social y psicológico y la nutrición implica lo biológico, social y ambiental. Estos avances en ambas áreas en las que el objetivo primordial de ambas es la salud colectiva e individual implica un compromiso para la formación de futuros profesionales tanto de la Educación para la Salud como de nutrición, que tengan la habilidad de observar a la nutrición como un elemento indispensable en la salud que permita el tener estilos de vida saludables para alcanzar una adecuada calidad de vida, en este sentido en México se están formando educadores para la salud profesionales capaces de incidir en los determinantes de la salud, dentro de estos se encuentra la nutrición con el objetivo de modificar actitudes, aptitudes y conductas que repercutan en adquirir estilos de vida saludables, disminuir enfermedades crónicas y así lograr una mejoría en la calidad de vida de la población.

Por otro lado el surgimiento de la educación nutricional como una disciplina enmarcada en la educación 
para la salud, se basa en el modelo biopsicosocial de la salud. De acuerdo a lo mencionado es una herramienta de la educación para la salud para promover una alimentación correcta y la adopción de hábitos alimentarios saludables, que significa la modificación de conductas y finalmente a prevenir enfermedades donde los hábitos inadecuados en materia de alimentación representen un factor de riesgo para desarrollarlas. Sin embargo la educación nutricional al estar vinculada con actividades de salud pública tiene un papel fundamental al diagnosticar los problemas alimentarios que aquejan tanto a los individuos como a las sociedades, considerando los aspectos de la tridimensionalidad ya explicados. Debido a que la educación para la salud tiene como objetivo primordial el promover estilos de vida saludables en las poblaciones, esto un implica un proceso de reaprendizaje que pretende que el individuo desaprenda los hábitos nocivos para su salud y reaprenda aquellos que son saludables; es decir; que las personas sean capaces de modificar sus conductas.

Para dejar en claro la articulación entre la tridimensionalidad de la nutrición y la educación para la salud se reduce a que ambas toman en consideración al individuo en su contexto, es decir, dejan de ser unidimensionales al no solo considerarlo desde una dimensión biológica sino también como un ser social y en constante relación con el medio. Por otro, mediante la educación nutricional lleva consigo un proceso de enseñanza - aprendizaje tal y como lo hace la educación para la salud, solo que el reto de esta última no solo implica el transmitir conocimientos en materia de nutrición, tiene además el de modificar conductas inadecuadas en materia de alimentación. Sin embargo el reto implica que se diseñen estrategias que involucren tanto el conocimiento como la modificación de conductas, para ello algunas alternativas son el adecuar juegos autóctonos de cada país a temas relacionados con la nutrición. Un ejemplo, en el caso de México consistiría en crear la lotería nutricional. Finalmente los profesionales de educación para la salud poseen todas las herramientas necesarias para abarcar las problemáticas de salud desde un punto de vista social sin perder de vista el biológico, por lo que tienen la responsabilidad de crear programas en materia de nutrición que promocionen y promuevan estilos de vida saludables en las poblaciones a través de conocimientos y modificaciones de conductas.

\section{RESUMEN}

Se describe la evolución del concepto de nutrición hasta llegar a la concepción de la tridimensionalidad del mismo. Analizándolo desde el nivel mundial, América Latina y México. Se consideraba a la nutrición como un proceso exclusivamente biológico, sin embargo actualmente involucra aspectos sociales y ecológicos. Se destaca la convergencia entre el concepto de salud y nutrición, a partir de la concepción biopsicosocial del individuo, motivo por el que se enfatiza la importancia de la educación para la salud como herramienta preventiva y promocional que no solo incluye el aspecto informativo sino también la modificación de conductas con el objetivo de que el individuo adquiera estilos de vida saludables. Se concluye con la manera como la educación para la salud se articula con las tres dimensiones de la nutrición.

Palabras clave: salud; promoción de la salud; enfermedades crónicas; nutrición comunitaria; educación nutricional.

Dirigir la correspondencia a:

Profesora

Adriana Ivette Macias Martínez

Unidad Académica Profesional Nezahualcóyotl

Universidad Autónoma del Estado de México.

Av. Bordo de Xochiaca s/n.

Colonia Benito Juárez, Nezahualcóyotl,

Estado de México. México.

Teléfono. 51126426

E-mail: adrivet_msmz@hotmail.com

\section{BIBLIOGRAFIA}

1. Cannon G. Leitzmann C. The new nutrition science project. Public Health Nutr. 2005; 8:787-794.

2. Pereira JM. Historia de la Nutrición. MEDSPAIN, España. 1999.

3. Beauman C. Cannon G. Elmadfa I. Glasauer P. Hoffmann I. et al. The Giessen Declaration. Public Health Nutr. 2005; 8:783-786

4. Beauman C. Cannon G. Elmadfa I. Glasauer P. Hoffmann I. et al. The principles, definition, and dimensions of the new nutrition science. Public Health Nutr. 2005; 8:695-698.

5. Bengoa JM. Algunos eslabones de su historia. $\mathrm{Nu}-$ trición en América Latina. SLAN, 2005, pp13-34.

6. Bourges H. Casanueva E. Reseña Histórica sobre la nutriología en México. Nutrición en América Latina. SLAN, 2005, pp. 174-216.

7. Gómez CC. De Cos BAI. Requerimientos nutricionales. Nutrición en atención primaria. Unidad de Nutrición Clínica y Dietética. Hospital Universitario La Paz, Madrid. España, 2001, pp. 15-26.

8. Pinto F JA. Carbajal AA. La dieta equilibrada, prudente o saludable. Nutrición y salud. Instituto de Salud Pública. Madrid, 2003, pp. 3-80.

9. Latham MC. Nutrición humana en mundo en desar- 
rollo. Organización de las Naciones Unidas para la Agricultura y la Alimentación (FAO). Colección FAO: Alimentación y nutrición $\mathrm{N}^{\circ} 29$. Roma, 2002, pp. 35-43.

10. López MC. Nutrición comunitaria. Nutrición en atención primaria. Unidad de Nutrición Clínica y Dietética. Hospital Universitario La Paz, Madrid. España, 2001, pp. 55-67.

11. Pettoello-Mantovani M. The social and environmental dimensions of nutrition science. Public Health Nutr 2005; 8:749-752.

12. Wahlqvist L. The new nutrition science: Sustainability and development. Public Health Nutr 2005; 8: 766-772.

13. Connor J. Loomis R. Ecología de los cultivos.
Mundi-Prensa Libros. España, 2002.

14. Leitzmann C. Wholesome nutrition: A suitable diet for the new nutrition science project. Public Health Nutr 2005; 8: 753-759.

15. Restrepo HE. Málaga H. Promoción de la salud: cómo construir vida saludable. Pan American Health Org 2001, pp. 24-56.

16. Perea QR. Bouché PH. Educación para la salud: (reto de nuestro tiempo). Ediciones Díaz Santos. España, 2004, pp. 3-18.

17. Valadez FI. Villaseñor FM. Alfaro AN. Educación para la salud: la importancia del concepto. Revista de Educación y Desarrollo, 2004, pp. 43-8.

18. Roque P. Educación para la Salud. Publicaciones Cultural. México, 2002, pp.195-208. 